\title{
Especulación, sentimiento y escisión en el joven Fichte. Análisis de sus Aforismos sobre religión y deismo
}

\author{
MARÍA JIMENA SOLÉ \\ CONICET - Universidad de Buenos Aires (Argentina) \\ jimenasole@ffilo.uba.ar
}

\begin{abstract}
Resumen
Este artículo propone un análisis de los Aforismos sobre religión y deismo escritos por J. G. Fichte en 1790, poco antes de su conversión a la filosofía kantiana. Sostenemos que este texto no expresa la adhesión de Fichte al determinismo, ni al punto de vista del corazón, ni tampoco se limita a presentar la religión y el deísmo como dos sistemas opuestos. Según nuestra interpretación, Fichte defiende dos tesis. En primer lugar, que la mezcla de ambos sistemas es inaceptable y hay que respetar el límite que existe entre ellos. En segundo lugar, que es imposible sostener ninguno de los dos sistemas, porque necesariamente entran en colisión y dejan al ser humano en una situación de desgarramiento. La conclusión del fragmento es, por lo tanto, la necesidad de buscar una posición superadora, que logre subsanar la escisión. Esa posición superadora será la Doctrina de la ciencia, que modifica los términos del planteo antagónico y logra la reconciliación de la libertad y la filosofía.
\end{abstract}

Palabra claves: Determinismo, libertad, Dios, razón, corazón, Filosofía.

\section{Speculation, feeling and scission in the young Fichte Analysis of his "Aphorisms on Religion and Deism"}

\begin{abstract}
This article proposes an analysis of the Aphorisms on Religion and Deism written by J. G. Fichte in 1790 , shortly before his conversion to the Kantian philosophy. We argue that this text does not express Fichte's accordance with determinism, or with the point of view of the heart, nor merely present religion and deism as two opposing systems. According to our interpretation, Fichte defends two theses. First, that the mixture of both systems is unacceptable and the limit between them must be respected. Secondly, that it is impossible to affirm neither of them, because they necessarily collide and leave the buman being in a situation of scission. The conclusion of the fragment is, therefore, the need to seek a position that overcomes this situation of scission. That position will be the Doctrine of science, which amends the antagonistic terms of the dilemma and achieves the reconciliation between freedom and philosophy.
\end{abstract}

Key words: Determinism, Freedom, God, Reason, Heart, Philosophy.

Doctora en Filosofía por la Universidad de Buenos Aires (2010). Es Investigadora Asistente del CONICET, Jefa de Trabajos Prácticos en la cátedra de Historia de la Filosofía Moderna (Departamento de Filosofía, FFyL, Universidad de Buenos Aires) y Profesora invitada en la Maestría en Filosofía de la Universidad Nacional de Quilmes. Entre sus publicaciones se destacan los libros: Spinoza en Alemania (1670-1789). Historia de la santificación de un Filósofo Maldito (2011) y El ocaso de la Ilustración: la polémica del spinozismo, selección de textos, traducción, estudio preliminar y notas de M. J. Solé (2013).

Recibido: 23/Mayo/2016 - Aceptado: 13/Julio/2016 
En una carta de 1795, usualmente citada en la bibliografía especializada, Fichte se proclama como el autor del "primer sistema de la libertad"1. Inspirado en la Revolución Francesa, que según escribe en esa misma carta, "ha roto las cadenas políticas del hombre", Fichte sostiene que su sistema, la Doctrina de la ciencia, en la teoría "arranca al hombre de las cadenas de la cosa en sí y de su influjo, que, en mayor o menor medida, lo ataban en todos los sistemas anteriores"; y en la práctica, le otorga fuerza para liberarse "por el ánimo sublime que transmite" (GA, III.2: 300). Así como los revolucionarios franceses lucharon contra el despotismo monárquico, Fichte construye su filosofía en pugna con otra posición filosófica que debe ser derrotada: el dogmatismo, al que caracteriza como materialista y determinista ${ }^{2}$.

Sin embargo, existen testimonios escritos que permiten sostener que durante su juventud, antes del descubrimiento de la Doctrina de la ciencia, Fichte adhirió al determinismo ${ }^{3}$. Incluso sabemos, a través del testimonio de Carl Fiedler, que hacia comienzos 1785 Fichte tenía la intención de escribir un ensayo sobre el fatalismo y la necesidad (GA, III.1: 9). Ese ensayo, sin embargo, nunca fue escrito. Recién en 1790 Fichte se ocupó del asunto del determinismo en un fragmento que fue publicado póstumo, titulado Aforismos sobre religión y deísmo ${ }^{4}$.

1 Citaremos las obras de Fichte según la edición crítica (1962 y ss.) con la abreviatura: $\mathrm{GA}=$ Gesamtausgabe der Bayerischen Akademie der Wissenschaften, indicando serie, tomo: página.

2 Así lo presenta, por ejemplo, muy claramente en la Primera Introducción a la Doctrina de la ciencia (1797). Véase GA, I.4: 188 y ss.; Traducción al español: Fichte, 1984.

3 Es posible reconstruir sus convicciones a partir de una serie de cartas enviadas a amigos cercanos en las que narra el abandono de estas convicciones, como efecto del impacto que generó en él la lectura de la Crítica de la razón práctica de Kant. En septiembre de 1790, Fichte escribe a su amigo Weisshun que desde que ha leído esa obra vive en un mundo nuevo, en el que principios que consideraba irrefutables se han venido abajo y en el que ha visto probadas cosas que creía imposibles de demostrar, entre ellas, el conceptos de una libertad absoluta y del deber (GA, III.1: 167). En otra carta de ese mismo mes, dirigida a su prometida Johanna Rahn, Fichte admite que sus disquisiciones acerca de la necesidad eran erróneas y le confiesa que en ese momento está "completamente convencido de que la voluntad humana es libre" (GA, III.1: 169 y 174). Poco después, en otra epístola dirigida a Achelis, Fichte escribe: "a usted especialmente debo reconocerle que ahora creo de todo corazón en la libertad del hombre" (GA, III.1: 193). Así pues, según sus propias palabras, antes de su encuentro con la segunda Crítica kantiana, Fichte había estado convencido de la imposibilidad de demostrar la libertad de la voluntad y, por lo tanto, también de la imposibilidad de fundar racionalmente una moral, basada en los conceptos de responsabilidad, deber y delito.

4 GA, II.1: 287-291. El texto fue publicado póstumo por el hijo de Fichte, Immanuel Hermann, por primera vez en 1831, en la segunda parte de un libro titulado Johann Gottlieb Fichte's Leben und litterarischer Briefwechsel (18-25). Luego en 1845, apareció en el tomo V de 
Este texto ha sido considerado por algunos especialistas como la expresión sistemática de esa postura determinista del joven Fichte. Así lo hace su propio hijo, Immanuel Hermann, editor de las Obras completas de su padre. En el prólogo al tomo V, que incluye ese fragmento, Immanuel H. Fichte hace referencia a las convicciones de su padre durante su juventud y sostiene que mientras era estudiante de teología en Leipzig, las enseñanzas de sus maestros lo habían llevado a buscar respuestas en la filosofía. "Fue entonces", escribe, "cuando la visión determinista del mundo le pareció como la más consecuente" (Fichte, 1845: vi). Y luego de indicar que Spinoza le sirvió como modelo para esa concepción filosófica, sostiene que la manera en que su padre comprendió el sistema determinista se encuentra en ciertos pasajes de los Aforismos sobre religión y deismo, que es el "testimonio de la etapa más temprana en la historia de su formación" (Fichte, 1845: vi).

En efecto, dado que Fichte hace referencia allí a la primera Crítica de Kant, pero no la segunda, se admite que escribió los Aforismos luego de su lectura de la Crítica de la razón pura, a finales de julio de 1790, y antes de la conversión motivada en él por la Crítica de la razón práctica, a finales de septiembre (GA, III.1: 167). Fichte tenía 28 años.

Dejando de lado la cuestión del spinozismo del joven Fichte -objeto de discusión entre los especialistas ${ }^{5}$ - muchos han aceptado que los Aforismos sobre religión y deísmo son la expresión de su determinismo ${ }^{6}$. Reiner Preul, quien ha abordado exhaustiva y sistemáticamente la época pre-kantiana del pensamiento de Fichte en una obra titulada Reflexion und Gefübl (1969), se ha opuesto a esa lectura, al señalar que si bien los especialistas se han concentrado en el aspecto determinista de los Aforismos... y han considerado que este texto expresa las convicciones del joven Fichte que la doctrina kantiana logra desterrar, se trata en realidad de una lectura simplista

las Sämtliche Werke de Fichte, también editadas por su hijo, que reúne escritos sobre filosofía y religión (1-8).

5 Que Spinoza haya sido el modelo a partir del cual Fichte construye la posición determinista es difícil de justificar a partir de la base textual. Quien primero discutió esa supuesta influencia fue W. Kabitz, quien sostuvo que la fuente de su posición determinista es Leibniz, a quien Fichte conocía a través de las obras de Crusius, Wolff o Platner (1902: 13 y ss.). Sin embargo, lo más probable es que Fichte haya tomado la doctrina determinista expuesta en sus Aforismos de K. F. Hommel, quien fue su profesor en la Universidad de Leipzig y en 1770 había publicado bajo el pseudónimo Alexander von Joch, un libro titulado Recompensa y castigo según las leyes turcas, donde rechaza el libre albedrío y expone una visión determinista basada en ciertos elementos de la filosofía de Leibniz (Nohl, 1911: 11ss.; Wildfeuer 1997b; Preul 1969: 121ss.).

6 Gueroult, por ejemplo, considera que ante el conflicto entre ambas facultades, Fichte se decide por la especulación (Gueroult, 1930: I,38). También afirman una posición similar, entre otros muchos especialistas, López Domínguez, 1995: 35; Ivaldo, 1996: 13.

VERITAS, No 35 (Septiembre 2016) 
del texto que no contempla el otro concepto presente en el fragmento, fundamental para el pensamiento fichteano y resultado de una larga meditación, esto es, el concepto de sentimiento o Gefübl (6 y ss. $)^{7}$. Así pues, Preul interpreta el fragmento como el esfuerzo de Fichte por reunir ambos puntos de vista -y ambas visiones del mundo- en una misma subjetividad, a pesar de que este intento revele la imposibilidad de que esa coexistencia sea armónica (Preul, 1969: 113ss.).

Años más tarde, contra la lectura de Preul, Wildfeuer ha reivindicado la interpretación según la cual los Aforismos... expresan el determinismo del joven Fichte, aunque éste no sería el sistema determinista tal como aparece en ese texto. En su obra Praktische Vernunft und System (1997), este autor sostiene que las dos posiciones presentadas en los Aforismos -el determinismo y la religión- presuponen un sistema de referencia único, que garantiza la posibilidad de que ambas coexistan. Ese sistema único es, según Wildfeuer, una forma del determinismo que, si bien no es mencionado explícitamente en el texto, debe ser presupuesto si se pretende comprender la propuesta fichteana de una religión que subsiste junto a un deísmo puro sin refutarse ni anularse mutuamente (Wildfeuer, 1997a: 164ss).

Frente a estas dos posiciones, surge en la bibliografía especializada una tercera propuesta: la de ver en el joven Fichte un defensor del sentimiento, de la certeza inmediata no racional basada en la constitución afectiva del ser humano. En esta línea, Vicente Serrano afirma en su investigación titulada Metafísica y filosofía transcendental en el primer Fichte (2004), que Fichte se revela en sus fragmentos tempranos como un lector de Rousseau, quien priorizaba el sentimiento sobre la especulación. Así pues, Serrano afirma que el verdadero problema para Fichte, ya desde el comienzo de sus meditaciones filosóficas, es el de encontrar la manera de justificar la prioridad de lo práctico por sobre lo teórico. Esta cuestión, que se encontraría expresada en otros textos anteriores, es también la preocupación central de los Aforismos (Serrano, 2004: 22-23).

Así pues, aunque mediante interpretaciones muy diferentes, tanto Preul como Wildfeuer leen el fragmento del joven Fichte como la exposición de dos sistemas opuestos que pueden darse en el mismo sujeto aunque Wildfeuer sostiene que para que eso sea posible, es necesario postular la existencia de un sistema de base determinista y Preul señala que el propio Fichte reconoce que su intento es en vano. Serrano, por el contrario, propone ver ya en los Aforismos la incipiente afirmación de una prioridad de la práctica por sobre la teoría, lo cual se traduce, según su lectura, en una adhesión por parte del joven Fichte al punto de vista del corazón.

En esta misma línea, Heimsoeth (1931: 25) sostiene que los Aforismos presentan frente a frente dos sistemas inconciliables. Véase también Carvalho, 2009: 84. 
Ante el desacuerdo existente entre los especialistas, el objetivo de este artículo es contribuir a la discusión, proponiendo otra interpretación de este fragmento. Tal como señala Preul y contra la lectura de Wildfeuer, creemos que los Aforismos no representan la declaración fichteana de adhesión al determinismo. Tampoco, como argumenta Serrano, representan su adhesión al punto de vista del sentimiento. Creemos que, efectivamente, el texto expone dos posiciones contrapuestas - la posición religiosa, que afirma la libertad de la voluntad, y la especulativa, que la niega- pero, según nuestra lectura y a diferencia de lo que sostiene Preul, no se limita al intento de presentarlos como dos sistemas que, basados en principios opuestos, son coherentes y no se contradicen. A través del análisis del texto, mostraremos que Fichte denuncia, en primer lugar y como señala bien Serrano, que la mezcla de ambos puntos de vista es inaceptable. Esa es, según nuestra interpretación, la primera tesis del fragmento. Pero hay una segunda tesis presente en el texto que nuestro análisis pondrá en evidencia: la imposibilidad de sostener ninguna de las dos posiciones - patentizada en la desesperante experiencia de la escisión-y, por consiguiente, la necesidad de buscar una posición superadora.

Así pues, argumentaremos en este artículo que los Aforismos sobre religión y deísmo no constituyen un texto aporético -como es en general leído (Preul, 1969: 121; Wildfeuer, 1997b: 64)-, sino que las preguntas que quedan abiertas y que no obtienen respuesta implican una toma de posición, expresan la necesidad de buscar un nuevo punto de vista, un sistema que no intente combinar religión y especulación, sino que logre subsanar la escisión en la que éstos abandonan al ser humano. Esa posición superadora resultará ser la Doctrina de la ciencia, que modifica los términos del planteo antagónico y supera la escisión a través de la reconciliación de la libertad y la filosofía: el primer sistema de la libertad.

\section{Religión y deísmo puro}

Los Aforismos sobre religión y deísmo se ocupan, en primer lugar, de investigar la naturaleza -la esencia- de la religión cristiana. Para ello, Fichte hace referencia a otras religiones, como la judía o el paganismo antiguo, con el fin de mostrar los elementos que ellas comparten así como sus rasgos específicos. Fichte dedica a este tema los primeros 12 aforismos, que representan aproximadamente dos tercios del texto.

La religión cristiana es presentada por Fichte como un sistema: un conjunto de proposiciones conectadas entre sí. Las proposiciones fundamentales del cristianismo presentan la peculiaridad de que "se presuponen 
como admitidas" (af. 1) y la investigación que conduciría a indagar sus posibles fundamentos se encuentra anulada. A partir de ellas "todo lo demás se sigue por medio de los más correctos argumentos, según la más clara conexión" (af. 2). La religión cristiana se basa, pues, en algunas proposiciones fundamentales -el conjunto de estas proposiciones, dice Fichte, constituirían su "canon" (af. 2) - que se aceptan sin intervención del razonamiento filosófico. "Si nos basamos en estas proposiciones", escribe, "todo en la religión parece tener la más clara coherencia" (af. 11). Se trata, pues, de un sistema correctamente confeccionado en el que la verdad de las proposiciones derivadas depende de la verdad -aceptada e imposible de poner en cuestión- de las proposiciones fundamentales.

Fichte pasa entonces a analizar el contenido de estas proposiciones fundamentales sobre las cuales se asienta la religión cristiana. Lo primero que advierte es que el cristianismo "considera a Dios únicamente en la medida en que Él puede tener relación con los seres humanos” (af. 3). No se ocupa de la esencia o de la existencia de Dios consideradas en sí mismas, en sentido "objetivo" -según la terminología que Fichte utiliza aquí y parece haber tomado de $\mathrm{Kant}{ }^{\prime}-$, sino que únicamente le interesa la divinidad en la medida en que se vincula con los hombres.

Este Dios para el hombre responde a una exigencia del corazón humano. Se trata del resultado de un requerimiento universal y, lo adelanta ya en el aforismo 4, se distingue del concepto de Dios al que se puede acceder mediante la especulación. Este Dios del corazón, para satisfacer esos requerimientos, debe poseer determinadas características. El corazón exige "un Dios al que se pueda implorar, que sienta compasión y amistad" (af. 4); el ser humano requiere "un Dios con el que pueda comunicarse, con el que sea posible establecer una relación de mutua transformación” (af. 4).

Frente a esos requerimientos, que como señala Fichte, son connaturales al hombre, a lo largo de la historia las religiones han apelado a diferentes estrategias. Fichte ofrece aquí, aunque de manera muy esquemática

8 Citaremos los Aforismos sobre religión y deísmo según nuestra traducción al español, que se incluye como apéndice a este artículo. Para facilitar la lectura, indicamos simplemente "af." y el número del aforismo.

9 Como se verá, los Aforismos parecen utilizar en sentido técnico la oposición entre validez objetiva y validez subjetiva. Mientras que Pruel sostiene que esto manifiesta la comprensión aún inmadura de Kant por parte de Fichte (1969: 108), Wildfeuer basa toda su interpretación en esta oposición, a la que le atribuye un valor hermenéutico fundamental (1997a: 176ss.). Por nuestra parte, consideramos que aquí se trata simplemente de oponer un conocimiento o una convicción basada en razones ("objetivo") frente a una convicción basada en sentimientos ("subjetiva"). En este sentido, se trata de conceptos que pueden ser remitidos a la doctrina kantiana, pero es claro que Fichte no los utiliza en su sentido estricto. 
y breve, una exploración del desarrollo histórico de la religión. El punto de quiebre en esta historia parece ser la aparición de la figura de Jesús. En efecto, Fichte indica que antes de la llegada de Jesús, las religiones "al comienzo más y luego gradualmente cada vez menos" (af. 5) apelaron al antropomorfismo para satisfacer esos requerimientos del corazón. También la religión judía es presentada como una de las religiones que atribuyen a su Dios características propias del ser humano. Esta estrategia, sin embargo, debió ser abandonada en la medida en que "la razón humana se elevó a un concepto consecuente de la divinidad" (af. 6). En un momento histórico dado, ya no fue posible pensar a Dios como un ser humano y, por lo tanto, se recurrió a una nueva estrategia que Fichte denomina "el sistema de la mediación" (af. 6). Se trata de la introducción de figuras que funcionan como mediadoras entre los hombres la divinidad: los penates o lares del paganismo, la figura de Jesús para el cristianismo y los santos del catolicismo, que surgieron "ya que luego de la venida de Jesús la humanidad volvió a hundirse" (af. 6 nota). Se trata, pues, de una historia de las religiones que culmina en el cristianismo como su resultado más elevado, a pesar de que linealmente, en su aparecer temporal, haya habido retrocesos, como el representado por el catolicismo papista.

Jesús es, pues, la figura que logra satisfacer los requerimientos del corazón de la mejor manera posible. A Jesús se le atribuyen "todas las propiedades de Dios que pueden referirse al ser humano" (af. 7) y también "aquellas propiedades que el corazón de los seres humanos buscan en su Dios, sin que el entendimiento las encuentre en él: compasión, amistad afectuosa, flexibilidad" (af. 8). Jesús es, al mismo tiempo, divino y humano; reúne en sí mismo ambas naturalezas.

La figura de Jesús se revela, inmediatamente, como fundamental no sólo para poder conceptualizar al Dios del corazón sino para la relación misma que se da entre Dios y el ser humano. Fichte sostiene que la proposición fundamental de todas las religiones, y también del cristianismo consiste en afirmar "que el pecado existe y que el pecador únicamente puede acercarse a Dios luego de determinadas conciliaciones" (af. 9). Nuevamente, se trata de una afirmación basada en el sentimiento, lo que Fichte denomina una Empfindungssatz. (af. 10) que no es demostrada racionalmente ni dice nada acerca de la realidad tal como es. Esta proposición hace referencia a la manera en que el corazón siente la situación del hombre y su relación con la divinidad: una situación de separación como resultado del pecado y la posibilidad de la reconciliación gracias a la muerte de Jesús. El sacrificio de Jesús puede ser pensado como propio por los cristianos que experimentan el requerimiento de una reconciliación (af. 10). El cristianismo presupone, por lo tanto, que los seres humanos son responsables de sus pecados y libres para buscar la conciliación con su creador. Ese 
concepto de libertad, de una voluntad que decide de manera incondicionada, también es, según Fichte, un concepto del sentimiento que la razón no puede demostrar (af. 15, nota).

De este modo, la religión cristiana se revela como un sistema coherente, basada en ciertas proposiciones fundamentales del sentimiento que la razón no puede investigar. Postula la existencia de un Dios creador misericordioso, flexible y amoroso. Postula también la existencia del pecado, pero introduce la posibilidad de que el ser humano -que es libre- se reconcilie con su creador. Esta conciliación es posibilitada a través del sacrificio de Jesús, mediador entre Dios y el ser humano. Es por ello que Fichte sintetiza el carácter propio del cristianismo del siguiente modo:

Estos primeros principios fundamentales de la religión se basan más en sentimientos que en convicciones: en el requerimiento de unirse con Dios, en la sentimiento de su caída en el pecado y su culpabilidad, etcétera. Así pues, la religión cristiana parece destinada más para el corazón que para el entendimiento. No pretende imponerse mediante demostraciones; quiere ser buscada por un requerimiento. Parece una religión de almas simples y buenas (af. 12).

Se trata, pues, de un conjunto coherente de proposiciones que se basan únicamente en el aspecto no especulativo sino afectivo del ser humano y que está destinado a las almas buenas y simples. Debe ser pensando, sin embargo, como universal, ya que las exigencias del sentimiento, sostiene Fichte, son compartidas por todos los seres humanos de todas las épocas.

Luego de clarificar la naturaleza de la religión cristiana, Fichte se ocupa del otro concepto que aparece en el título del texto: el deísmo. A ello dedica principalmente los aforismos 15 y 16. Si se dejan de lado las exigencias del corazón y se procede únicamente con la reflexión, sostiene Fichte, de manera consecuente y sin preocuparse por los resultados de esta investigación, entonces necesariamente se arriba a las siguientes afirmaciones:

a) Hay un ser eterno, cuya existencia y cuya manera de existir es necesaria.

b) Según y a través del pensamiento eterno y necesario, este ser produjo el mundo.

c) Cada cambio en este mundo es determinado por una causa suficiente necesariamente tal como es. La primera causa de cada cambio es el pensamiento originario de la divinidad.

d) Asimismo, cada ser pensante y sintiente debe existir necesariamente del modo en que existe. Ni su actuar ni su padecer pueden ser de otra manera que como son, sin contradicción. 
e) Lo que el sentimiento humano universal llama pecado, es producido por la limitación necesaria, mayor o menor, de los seres finitos. Se dan efectos necesarios en la situación de estos seres, que son tan necesarios como la existencia de Dios e igualmente imposibles de eliminar (af. 15).

Se trata del sistema que Fichte denomina "sistema del puro deísmo" (af. 16) y cuya proposición fundamental es el principio de razón suficiente que establece que todo lo que sucede tiene una causa eficiente que da cuenta de su existencia y de la manera determinada en que existe -explicitada en el punto (c) del aforismo que acabamos de citar-. Nada queda sin explicación. Todo tiene una causa. Dios, su pensamiento, es identificado como la primera causa de todo lo existente en el mundo. Es, por lo tanto, un ser eterno que existe también necesariamente de una determinada manera.

Ahora bien, este Dios demostrado racionalmente presenta grandes diferencias respecto del Dios del corazón. Ya en el Aforismo 4 Fichte menciona esta diferencia. Mientras que el corazón exige un Dios que escuche las plegarias de los hombres, que se compadezca de sus males y que tuerza su voluntad en función de ellos, la especulación le ofrece un Dios "como un ser inmutable, incapaz de cualquier pasión" (af. 4), una divinidad "que no tiene ningún punto de contacto (...) con ningún ser finito" (af. 4).

Este Dios de la razón produce un universo en el que reina el determinismo. Los seres finitos del mundo, pensantes y sintientes, también poseen una existencia necesaria. Así, tanto la posibilidad de una libertad de la voluntad, como la posibilidad de que el orden universal establecido pueda modificarse, quedan totalmente descartadas. Todo lo que sucede es un efecto necesario de una causa, que a su vez es efecto necesario de otra causa y así indefinidamente. Todos los hechos del mundo son resultado de una necesidad universal imposible de modificar. En este contexto, el pecado o el delito como hecho atribuible a una voluntad libre individual es impensable. En un sistema del puro deísmo, lo que usualmente es considerado como pecado o falta, no es más que limitación; una limitación que es propia de todo ser finito, inevitable. Así, la especulación ofrece una visión del mundo que afirma de manera radical el determinismo universal y excluye la atribución de valores morales a los acontecimientos humanos o naturales.

Fichte no denuncia este sistema deísta puro como nocivo. De hecho, según él, "no tiene ninguna influencia perjudicial" (af. 16) para la moral. Lo único que hace, reconoce el autor, es impedir que el hombre tome parte de esos sentimientos agradables que produce la religión y, en cambio, genera cierta "inflexibilidad" (af. 16). 
Como ya mencionamos, es importante señalar que esta posición que Fichte denomina "sistema del puro deísmo", no coincide con el deísmo entendido como la posición teológica ${ }^{10}$. Como se sabe, el deísmo -impulsado en Inglaterra por Locke, Hume y Tindal, y en Francia por Rousseau y Voltaire- sostiene que los principios fundamentales de la religión, como la existencia de Dios entendido como un creador inteligente y bueno, la inmortalidad del alma y la eternidad de las penas, pueden ser demostrados racionalmente. Plenamente fundado en la razón, sin necesidad de recurrir a ninguna otra fuente de verdad, el deísmo propone la instauración de una religión natural, que coincidiría en definitiva con la moral, y que cada ser humano podría conocer por sí mismo sin necesidad de revelación sobrenatural, sacerdotes, templos, ritos o ceremonias. En Alemania, esta posición fue adoptada por muchos hombres de la Ilustración, como por ejemplo Moses Mendelssohn, Samuel H. Reimarus y Carl F. Bahrdt ${ }^{11}$.

El único punto en común entre esta posición deísta y lo que Fichte denomina "sistema puramente deísta" consiste -tal como lo señala Preul (1969: 110) - en la identificación de la causa primera del universo con una inteligencia suprema. Sin embargo, el sistema del deísmo puro expuesto por Fichte excluye tanto la libertad humana como la existencia de valores morales y por lo tanto anula la posibilidad de fundar una moral o una religión natural sobre la base de principios racionales. En este sentido, se identifica en realidad con el "razonamiento filosófico" (af. 2).

\section{Sentimiento o especulación}

La investigación acerca de la naturaleza de la religión cristiana y del deísmo puro revela una contraposición fundamental que remite en realidad a dos dimensiones propias de la naturaleza humana, a dos facultades diferentes, presentes en todo ser humano. Por un lado, la dimensión sentimental, que remite a lo que Fichte llama el sentimiento universal (allgemein Empfindung) o el corazón (Hery). Por el otro, la dimensión racional, que

10 Acerca del concepto de deísmo, véanse Allison, 1966; Carvalho, 2009: 16ss.; Albrecht-Engel, 1994; Gawlick, 2001.

11 S. H. Reimarus fue uno de los representantes más radicales de esta corriente. Luego de su muerte, entre 1774 y 1778, G. E. Lessing seleccionó partes de una de sus obras inéditas, la Apología o escrito en defensa de la veneración racional de Dios [Apologie oder Schutzschrift für die vernünftigen Verehrer Gottes], y las publicó como una serie de textos conocidos como los Fragmentos de un anónimo. Estos Fragmentos reivindican el rol de la razón como única fuente de verdad y, contra la religión histórica, argumentan que las creencias de las personas son el resultado de prejuicios transmitidos en su infancia. Además, mediante una exégesis racional del Antiguo y del Nuevo Testamento, ponen en evidencia que el contenido de los textos sagrados no puede ser considerado verdadero y llegan incluso a denunciar la religión cristiana como un fraude. 
Fichte denomina indistintamente especulación (Especulation) o reflexión (Nachdenken).

Como vimos, el sentimiento provoca ciertos requerimientos en los seres humanos, exigencias prácticas que deben ser satisfechas. Sus proposiciones, sus afirmaciones y principios tienen, pues, como único objetivo la satisfacción de esos requerimientos. Fichte habla del sentimiento universal, de modo que no sea confundido con los sentimientos privados, individuales de cada ser humano. Se trata, pues, de un aspecto constitutivo de todo ser humano y es por eso que sus afirmaciones poseen validez universal, aunque sea meramente una "validez subjetiva" (af. 16). El resultado sistemático que satisface a estos requerimientos es la religión, y la que mejor lo hace es la religión cristiana.

La especulación o reflexión también es presentada como una facultad común a todos los seres humanos, que investiga las esencias y las existencias de sus objetos tal como son en sí mismos -en el sentido que Fichte llama aquí "objetivo" (af. 3 y 7)- dejando de lado cualquier exigencia del corazón. El sistema que se construye sobre la base de los principios del conocimiento humano y mediante la deducción rigurosa de sus consecuencias es, pues, la filosofía.

Sentimiento y especulación son dos facultades humanas que no tienen nada en común, que producen edificios sistemáticos totalmente heterogéneos y que, por lo tanto, no deberían entrar en conflicto. Por un lado, las exigencias del corazón excluyen la posibilidad de una indagación racional acerca de sus afirmaciones. "Parece que es un requerimiento universal para el ser humano, buscar en su Dios ciertas características que le impidan el ingreso al ámbito de la especulación" (af. 4), escribe Fichte. En este sentido advierte, en diferentes momentos del texto, que las investigaciones se interrumpen respecto del ser o la esencia "objetiva" (af. 3) del Dios cristiano, así como acerca del pecado y de la libertad de la voluntad (af. 15 nota). La existencia de una divinidad tal como el sentimiento la requiere, la existencia del pecado y la posibilidad de la conciliación, así como la libertad humana, son afirmaciones que se basan únicamente en el sentimiento y no pueden ser demostradas. Por el otro lado, el sistema puramente deísta, sostiene Fichte, "no contradice a la religión cristiana, sino que le deja toda su validez subjetiva" (af. 16); no la falsea, "ya que en ningún lugar entra en colisión con ella" y tampoco se opone "a que se la venere como la mejor religión popular, ni a que sea recomendada con la más sincera efusión a aquellos que la requieren" (af. 16). La especulación no confirma ni refuta a la religión cristiana, pues sus afirmaciones no se basan en las "proposiciones fundamentales del conocimiento humano" (af. 15, nota), que son los únicos principios con los que ella cuenta. Existe, según 
Fichte, un limite (Gränze) que separa ambas facultades y que debe ser respetado:

Es notable que en los primeros siglos, apóstoles iletrados hayan suspendido sus investigaciones exactamente en el mismo sitio donde el pensador más grande del siglo XVIII, Kant, seguramente sin tener esto en cuenta, establece el límite de la investigación acerca de la esencia objetiva de Dios, de la investigación de la libertad, la imputación, la culpa y la pena (af. 13).

Fichte se admira de que el límite al que la especulación debe atenerse haya sido señalado por los apóstoles y por el máximo filósofo de la época, Immanuel Kant. Como se sabe, la Crítica de la razón pura sostiene la imposibilidad de conocer lo suprasensible, lo que no puede darse en la experiencia, pues los objetos del conocimiento son el resultado de la aplicación de las categorías del entendimiento al fenómeno provisto por la sensibilidad, que consiste, a su vez, en el múltiple sensible dado, conformado bajo las formas puras del espacio y el tiempo. Todo aquello que se encuentra en el ámbito de lo suprasensible jamás puede devenir objeto del conocimiento, aunque Kant admita la posibilidad de pensarlo. En este sentido, las ideas de Dios, el alma y el mundo pueden ser en Kant pensadas por la razón pero jamás conocidas como objetos de la ciencia.

Religión y deísmo puro, el sistema basado en el corazón y el sistema basado en la especulación, son posiciones contrapuestas, pero -al menos en principio- no se excluyen mutuamente. Sentimiento y reflexión son facultades sin nada en común, pero no son presentadas por Fichte como facultades en pugna. Cada una posee su territorio. No se niegan, no se refutan, no se falsean la una a la otra.

Ahora bien, la total heterogeneidad que existe entre el sentimiento y la especulación no anula la posibilidad de la mezcla. Reconocer el límite no implica que éste sea respetado siempre. En efecto, lo que señalamos como la posición de Fichte en este fragmento consiste precisamente en denunciar la mezcla de ambos punto de vista como inaceptable. Fichte dedica el aforismo 14 y la nota al aforismo 15 a examinar los casos en los que se entremezclan ambos puntos de vista. Es llamativo, sin embargo, que a pesar de que al plantear la existencia del límite, Fichte habla desde el costado de la especulación, señalando que tanto los apóstoles como Kant se abstienen de llevar sus investigaciones más allá del uso legítimo de su razón especulativa, en el momento de abordar concretamente la posibilidad de que el límite se traspasado, se ocupa únicamente de la situación inversa, en la que el corazón impide el desarrollo de la especulación. Esta situación se da, según Fichte, de dos maneras diferentes. 
En primer lugar, el corazón se inmiscuye en la marcha de la reflexión, cuando impone las conclusiones a las que se quiere llegar:

Cuando se sobrepasa este límite, sin dejarle a la investigación el camino libre; cuando al comenzar a pensar se pone ya en la mira el objetivo al que se quiere llegar con el fin de que, en la medida de lo posible, la especulación y las afirmaciones de la religión coincidan, entonces el resultado es una casa construida sobre el aire con materiales muy diversos y muy mal unidos (af. 14).

Fichte ofrece aquí dos ejemplos de esta situación, que remiten a posiciones asumidas por sus contemporáneos en el ámbito de la teología. El primer ejemplo es lo que él denomina la "filosofía religiosa" que se encuentra en las obras de Christian August Crusius, a quien califica como un pensador "cobarde y dotado de escasa fantasía". Crusius (1715-1775) fue profesor de Teología en la Universidad de Leipzig, donde el propio Fichte estudió, y sus obras tuvieron cierta popularidad en su época. Pasó a la historia como uno de los más sagaces enemigos de la filosofía de Leibniz y Wolff. Principalmente, criticó su adhesión irrestricta al principio de razón suficiente y el consecuente determinismo que de allí se sigue. Según Crusius, por el contrario, la voluntad es libre y en el ámbito de la teología reivindicó la posibilidad de unificar la revelación y la razón. El segundo ejemplo al que hace referencia Fichte apela a "los nuevos teólogos", a quienes considera "más valientes y más ocurrentes" que a Crusius, autores de una "religión filosófica, o un deísmo" (af. 14). El término deísmo aparece aquí tomado en su sentido habitual, y no debe confundirse con lo que Fichte llama "el sistema puramente deísta" (af. 16) que, como vimos más arriba, es la posición estrictamente racional, basada únicamente en los principios del conocimiento humano, sin mezcla del sentimiento. El deísmo, tomado como una religión filosófica, es denunciado en este pasaje como un castillo en el aire que surge del intento de justificar demostrativamente, mediante argumentos racionales, las exigencias del corazón. Se trata, pues, de una construcción incoherente, frágil y engañosa.

Estos dos casos, el de una religión filosófica -defendida por los "nuevos teólogos" o los "deístas"- y una filosofía religiosa -representada por la posición híbrida de Crusius- son rechazados por ser el resultado de la imposición de las exigencias del corazón sobre la razón, que se ve obligada a armonizar sus descubrimientos con esas verdades del sentimiento, admitidas pero no justificadas ${ }^{12}$.

12 Estas críticas pueden aplicarse también a la otra corriente teológica que era dominante hacia finales del siglo XVIII en Alemania: la llamada neología. Como una reacción frente al deísmo, y también frente al ateísmo de los materialistas franceses, esta corriente 
Existe, además, una segunda situación en la que el corazón puede obstaculizar el ejercicio de la reflexión recurriendo a otra estrategia: la de establecer la proposición fundamental a partir de la cual se deduce todo lo demás, y hacerla pasar por una afirmación justificada especulativamente. Fichte denuncia a los filósofos que pretenden demostrar todo de manera rigurosa, pero que frecuentemente "en las cadenas demostrativas se guardan para sí mismos sus conclusiones y comienzan una nueva cadena con nuevos principios, que han encontrado en alguna otra parte" (af. 15, nota). El ejemplo que ofrece remite al "defensor de la libertad en las antinomias kantianas", quien según Fichte "había obtenido el concepto de la libertad de alguna otra parte (del sentimiento, sin duda) y que, en sus pruebas, no hace sino justificarlo y aclararlo" (af. 15, nota). Como es evidente, la referencia es a la tercera antinomia de la Crítica de la razón pura de Kant, en la que se afirma como tesis que la causalidad según leyes naturales no es suficiente para explicar los fenómenos del mundo, sino que para ello hace falta también la "causalidad por libertad" (407; A 445/B 473). La antítesis, por su parte, afirma que "no hay libertad" y que todo sucede "exclusivamente según leyes de la naturaleza". Fichte sostiene que la única fuente posible para la afirmación de la libertad es el sentimiento y que el defensor de la tesis de esta antinomia incurre en engaño al intentar hacerla pasar por una proposición demostrada racionalmente. En efecto, el desarrollo anterior del texto había puesto en evidencia la imposibilidad de afirmar la libertad de la voluntad mediante la sola reflexión: "en las cadenas argumentativas no modificadas que se siguen de las proposiciones fundamentales del conocimiento humano", sigue Fichte, "jamás hubiese llegado a un concepto de esa clase" (af. 15, nota). Un sistema filosófico que afirme la libertad de la voluntad no es más que otro castillo en el aire.

\section{La escisión}

La religión se basa en el sentimiento. La filosofía -o sistema del deísmo puro- se basa en la especulación. Ambos sistemas no deben mezclarse, si no se quiere incurrir en incoherencias y dar lugar a edificios teó-

representa un esfuerzo por reunir el cristianismo con la Ilustración. En efecto, la neología coincide con el deísmo en la postulación de la necesidad de que la religión sea racional, pero a diferencia de aquél, no rechaza la revelación sino que se propone racionalizarla. Así pues, la neología fue la posición teológica defendida por las grandes personalidades de la Ilustración alemana, como Ernesti, Sack, Spalding, Jerusalem y Semler, quienes sostuvieron la posibilidad de una completa racionalización del contenido de la revelación cristiana mediante el examen filosófico, histórico y filológico de la doctrina. Al respecto, véanse Allison, 1966; Carvalho, 2009: 16 ss.; Albrecht-Engel, 1994; Sparn, 2001. 
ricos engañosos. Fichte se esfuerza, como vimos recién, en señalar la necesidad de reconocer y respetar el límite que se alza entre sentimiento y corazón. La posibilidad de que sus resultados o sus puntos de partida coincidan, queda totalmente excluida.

Ahora bien, que exista un límite, que la diferencia entre ambas facultades sea reconocida, que se señale la necesidad de no mezclarlas si se quiere edificar sistemas coherentes, que se reconozca incluso el hecho de que el sistema filosófico no niega ni refuta al del corazón, no significa que el límite pueda ser respetado siempre. Esto es lo que, según nuestra lectura, el final del fragmento pone de manifiesto: el sistema de la religión y el sistema del puro deísmo, cada uno con sus proposiciones fundamentales, cada uno con sus conclusiones rigurosamente encadenadas, sin mezcla, sin puntos de contacto, no pueden permanecer uno frente a otro, simultáneamente, como dos visiones contrapuestas de la realidad ${ }^{13}$.

Como ya vimos, por un lado, la religión impide a la razón avanzar sobre sus principios fundamentales. Por otro lado, el ser humano que permanece en el punto de vista especulativo experimenta una inflexibilidad, una rigidez que le impide disfrutar del consuelo que la religión ofrece a las almas simples y buenas. Esta situación de mutua limitación no puede perdurar en el tiempo, porque tanto el corazón como la reflexión pretenden ir más allá y reclamar su derecho sobre los objetos del otro.

Los últimos dos aforismos con los que concluye el fragmento ponen en evidencia que esta contraposición entre sentimiento y reflexión, entre corazón y especulación, entre religión y filosofía, introduce en el ser humano una escisión que se revela insoportable:

Sin embargo, puede haber ciertos instantes, en los que el corazón toma venganza de la especulación, en los que se dirige con un anhelo ardiente al Dios reconocido como carente de compasión, como si Él fuese a modificar su gran plan a causa de un individuo. Puede haber ciertos instantes en los que el sentimiento de una ayuda visible, de una atención casi incuestionable a las plegarias, destruye todo el sistema; y cuando se extiende el sentimiento del desagrado de Dios frente al pecado, puede haber instantes en los que surge el anhelo apremiante de una reconciliación (af. 17).

La especulación puede construir un firme edificio sistemático, en el que se demuestra todo de manera rigurosa sin aceptar nada que no esté

13 Este punto de vista es esbozado por Carvalho, quien sostiene que junto con la exposición del determinismo y la posición del corazón, lo más significativo de los Aforismos es "la subsistencia de un residuo de resistencia a estas concepciones" (Carvalho, 2009: 85). Aquí llevamos esta sugerencia más lejos. Creemos que la resistencia a estas visiones no es residual sino que constituye la tesis principal del texto.

VERITAS, No 35 (Septiembre 2016) 
fundado sobre los principios supremos del conocimiento humano. El ser humano que especula vive en un mundo en el que rige el determinismo universal, en el que Dios es la causa necesaria, eterna e inmutable del universo, en el que todos los eventos presentes, pasados y futuros tienen una explicación causal. Sin embargo, los resultados de esa construcción racional son tan contarios a las exigencias del corazón, que éste irrumpe en ese mundo racional para aniquilarlo en un solo instante. Por eso Fichte habla de la venganza del corazón, que se enfurece al ver que la razón no satisface sus anhelos.

No importa cuán riguroso sea el razonamiento, habrá momentos de penuria, de duda, en los que el sentimiento obligará al filósofo a traicionar sus certezas, obligándolo a rezarle al Dios inmutable, a interpretar los eventos necesarios de la naturaleza como si fuesen la obra contingente de un creador bueno, a creer que hemos torcido el curso de los acontecimientos gracias a las plegarias, a sentir culpa frente a las propias limitaciones y desear la reconciliación con una divinidad paternal, buena, compasiva.

El ser humano experimenta de este modo un desgarramiento en su propio ser, donde conviven, se superponen y se enfrentan, las exigencias del corazón y las enseñanzas de la reflexión. El último aforismo deja en claro la imposibilidad de que ambos sistemas subsistan:

¿Cómo hay que tratar con un ser humano semejante? En el campo de la especulación parece imbatible. No hay que ofrecerle pruebas de la verdad de la religión cristiana. Pues las admite únicamente en la medida en que es posible demostrárselas, pero alega que le es imposible recurrir a ellas. Es capaz de comprender las ventajas que rehúye de esta manera. Puede desearlas con el anhelo más ardiente, pero le es imposible creer (af. 18).

Todo ser humano siente pero también, en mayor o menor medida, especula. En cuanto que especula correctamente, es inapelable, sus conclusiones son irrefutables, e incluso comprende las ventajas propias de la religión que su punto de vista racional lo obligan a rechazar. En cuanto que siente, desea esas ventajas, quiere unirse a la divinidad, experimenta su libertad y quiere ser perdonado por sus pecados. Cuando siente, cancela la especulación. Cuando especula, debe acallar al corazón. ¿Qué se hace con un ser humano semejante? Fichte parece esbozar, en las últimas líneas, una vía de escape:

El único medio de salvación para él consistiría en llevar sus especulaciones más allá del límite. ¿Pero es capaz de hacerlo cuando quiera? ¿Es capaz de hacerlo, en la medida en que se le ofrece la prueba irrebatible del carácter engañoso de estas especulaciones? ¿Puede hacerlo, en la medida en que este 
modo de pensar se encuentra entrelazado con él, ya sea por naturaleza, ya con el sesgo completo de su espíritu? (af. 18).

El corazón humano no puede ser acallado, tampoco ignorado. Pero el ser humano no pretende solo sentir, sino también conocer. El modo de pensar especulativo se encuentra entrelazado con él. Por lo tanto, si no se quiere permanecer en la escisión -y efectivamente la escisión es inhabitable, no ofrece salvación ni a creyentes ni a filósofos-, hay que abandonar tanto la religión cristiana como el sistema del deísmo puro. La única vía de escape, se atreve a escribir Fichte, consiste sería ir con la razón más allá del límite. Intentar demostrar aquello que el corazón exige. Llevar la investigación más allá de la frontera trazada por la religión.

El final del texto adquiere un tono desesperado en la repetición de una pregunta: ¿puede el ser humano reflexionar más allá del límite? Fichte no ofrece ninguna respuesta. Sin embargo, la pregunta implica una posición tomada: los sistemas contrapuestos no pueden sostenerse uno frente a otro, el conflicto entre ellos es inevitable e introduce un desgarramiento en el ser humano que evidencia la necesidad de abandonarlos y buscar otro horizonte.

\section{La Doctrina de la ciencia como reconciliación}

Tal como creemos haber mostrado suficientemente mediante el análisis del texto, Fichte no se inclina por ninguna de las dos visiones contrapuestas que presenta en los Aforismos. En este momento de su vida, no es ni un determinista convencido ni un defensor de la religión del corazón. Su posición parece limitarse, en un primer momento, a señalar que el ser humano está constituido de tal manera que tiene sentimientos y tiene también la capacidad de reflexionar, de argumentar racionalmente. En la medida en que atiende a los requerimientos de su corazón, no puede sino afirmar la existencia de un Dios bueno, caritativo, compasivo, que puede modificar su voluntad para atender a sus plegarias; sostiene, además, la existencia del pecado y la posibilidad de volver a reunirse con la divinidad -reconciliación que, en el cristianismo, se logra a través de la muerte de Jesús; y afirma la libertad de su voluntad y, por lo tanto, atribuye el pecado y la reconciliación exclusivamente a la responsabilidad moral de cada individuo. Pero en la medida en que especula, el ser humano se pregunta por la existencia y la esencia objetivas de Dios -quiere conocer a Dios tal como es en sí mismo y no únicamente en relación al ser humano- y reconoce que Dios es un ser eterno que existe necesariamente y que produce un universo en el que todo existe también necesariamente de una cierta ma- 
nera y no de otra. Esta afirmación de la necesidad universal anula la existencia del libre arbitrio y del pecado, transformándolo sencillamente en la limitación propia de todo lo finito. A partir de la razón especulativa es imposible, pues, fundar una religión, tampoco una moral. Sin embargo, el resultado de este desarrollo conduce a poner en evidencia que esta contraposición entre especulación y corazón introduce una escisión en el ser humano que es vivida con angustia y que pone en evidencia la imposibilidad de mantenerse en esa duplicidad.

Que esta situación de desgarramiento era experimentada en ese momento por el propio Fichte se sigue de lo que él mismo confiesa en una de las famosas cartas en las que relata su conversión al kantismo, dirigida a su amigo Heinrich Nikolaus Achelis en noviembre de 1790:

Me arrojé a la filosofía y en particular, como es obvio, a la filosofía kantiana. Allí encontré el antídoto para la verdadera fuente de mi mal y, por añadidura, suficiente alegría. La influencia que esta filosofía, en particular la parte moral de la misma -que es incomprensible sin el estudio de la Crítica de la razón pura- tiene sobre todo el sistema de pensamiento de un hombre, la revolución que gracias a ella se ha producido en todo mi modo de pensar, es inconcebible. A usted debo reconocerle especialmente, que ahora creo de todo corazón en la libertad del hombre y comprendo claramente que sólo bajo esta presuposición son posibles el deber, la virtud y, en general, la moral. Se trata de una verdad, que también yo admitía desde hace tiempo, a pesar de que, como quizás también se lo confesé a usted alguna vez, forzado por la totalidad de los resultados de mis argumentaciones, rechazaba toda la moral (GA, III.1, 193).

Fichte admite aquí haber experimentado la escisión en la que necesariamente es abandonado el ser humano cuando corazón y especulación son consideradas como dos facultades opuestas que no pueden ni deben combinarse. Mientras que su corazón le ensañaba que él mismo era libre, su razón especulativa lo conducía al determinismo. Fichte experimentaba entonces la urgencia de dejar atrás la escisión. Pocos años más tarde, escribirá que Doctrina de la ciencia era un requerimiento para él -y que pretende que también lo sea para sus contemporáneos, a quienes no tiene intención de imponer su filosofía ${ }^{14}$.

Efectivamente, si bien los Aforismos no hacen -no pueden hacer- referencia a lo que será el sistema fichteano, sí expresan la dirección que hay

14 "La Doctrina de la ciencia no debe imponerse en absoluto, sino que debe ser una necesidad, como lo era para su autor" (Fichte, 1984: 9), escribe Fichte en el prefacio a la Primera Introducción a la Doctrina de la ciencia. La palabra que J. C. Cruz traduce por necesidad es, en realidad, Bedürfnis que remite a la noción de algo requerido, de una necesidad práctica o una exigencia para la vida. 
que tomar para escapar a la disyuntiva entre el corazón y la reflexión, entre la visión religiosa que afirma la libertad humana y la visión filosófica del mundo que afirma un determinismo universal. Se trata de ir con la especulación más allá de lo que parecía ser un límite infranqueable. Ante la disyuntiva excluyente entre una visión sentimental del mundo que afirma la libertad y la existencia de un Dios moral, y una visión racional del mundo que postula el determinismo universal y anula así todo fundamento de una vida práctica, Fichte se propone reconciliar la libertad con la razón y producir el primer sistema de la libertad.

Como se sabe, fue Kant quien permitió a Fichte acceder al punto de vista superador, gracias a un concepto de razón como una facultad compleja que no se agota en su uso teórico sino que tiene también un uso práctico. Fue Kant quien le enseñó que el concepto de la libertad podía ser demostrado por la razón práctica. ${ }^{15}$ Sin embargo, lejos de volver a caer en un planteo dicotómico -que el propio Kant intentará subsanar en su Crítica del juicio - entre un mundo natural configurado por la razón teórica y un mundo moral propio de la razón práctica, Fichte sostiene la necesidad de que haya un único principio fundamental, a partir del cual se edifica todo el sistema. La Doctrina de la ciencia surge entonces como el sistema que logra reconciliar las exigencias del corazón y los principios de la reflexión, pero no mediante la combinación o la mezcla de ambas -cosa que los Aforismos rechazan claramente- ni tampoco mediante el intento de que coexistan pacíficamente, sino a través de un movimiento de elevación, que sitúa al filósofo en un punto de vista diferente: el momento originario, fundante de todo lo real, tanto de la naturaleza como del espíritu ${ }^{16}$. Ese primer principio fundamental de la Doctrina de la ciencia es un Yo absoluto. Un Yo cuya

15 "El concepto de la libertad, en cuanto su realidad queda demostrada por medio de una ley apodíctica de la razón práctica, constituye la piedra angular de todo el edificio de un sistema de la razón pura, incluso la especulativa, y todos los demás conceptos (los de Dios y la inmortalidad) que, como meras ideas, permanecen sin apoyo en la razón especulativa, se enlazan con él y adquieren con él y por él consistencia y realidad objetiva, es decir, que su posibilidad queda demostrada por el hecho de que la libertad es real; pues esta idea se manifiesta por medio de la ley moral" (Kant, 1951: 9).

16 "Pero nuestro sistema añade una parte práctica que fundamenta y determina a la primera [la parte teórica], acaba así toda la ciencia y agota todo lo comprendido en el espíritu humano; de este modo el entendimiento humano común (den gemeinen Menschenverstand), vilipendiado por toda la filosofía pre-kantiana, separado al parecer de la filosofía por nuestro sistema teórico sin la menor esperanza de reconciliación (Versöhnung), será otra vez reconciliado (wieder aussöhnt) con ella" (Fichte, 1975: 34). Así se refiere Fichte su sistema en 1794, en su primera exposición pública de la Doctrina de la ciencia ante los estudiantes de la Universidad de Jena, en el contexto de la discusión con el sistema de Spinoza a quien considera, precisamente, como el mejor representante de la posición dogmática y determinista. Así pues, a pesar de que Spinoza no es mencionado en los Aforismos, poco tiempo después, en 1794 -luego de que Fichte lee y estudia a Spinoza

VERITAS, No 35 (Septiembre 2016) 
esencia consiste en ponerse a sí mismo, que no es más que la actividad de afirmarse como actuante, que fundamenta tanto el saber como el actuar y que, en definitiva, no es sino pura libertad ${ }^{17}$.

\section{REFERENCIAS}

-Albrecht, M. \& Engel, E. J. (Eds.) (1994). Moses Mendelssohn und die Kreise seiner Wirksamkeit. Tübingen: Niemeyer.

-Allison, H. (1966). Lessing and the Enlightenment. Michigan: University of Michigan Press.

-Carvalho, M. J. (2009). "Introducción histórica”. En J. Rivera de Rosales y O. Cubo (Eds.), La polémica sobre el ateísmo, Fichte y su época (pp. 13-147). Madrid: Dykinson.

-Fichte, J. G. (1962 y ss.) [GA]. Gesamtausgabe der Bayerischen Akademie der Wissenschaften (40 Tomos, R. Lauth, E. Fuchs y H. Gliwitzky, Ed.). Stuttgart-Bad Cannstatt: Frommann-Holzboog.

-Fichte, J. G. (1845). Sämtliche Werke (Tomo V., Immanuel H. Fichte, Ed.). Berlin: Verlag von Veit und Comp.

-Fichte, J. G. (1831). Johann Gottlieb Fichte's Leben und litterarischer Briefwechsel II. Sulzbach: Seidelschen Buchhandlung.

-Fichte, J. G. (1984). Primera y Segunda introducción en la Doctrina de la Ciencia (J. Gaos, Trad.). Madrid: Sarpe.

-Fichte, J. G. (1975). Fundamento de toda la Doctrina de la Ciencia (J. Cruz Cruz, Trad.). Buenos Aires: Aguilar.

-Gawlick, G. (2001). "Deismus". En W. Schneiders (Ed.), Lexicon der Aufklärung (pp. 81-82). München: Beck.

-Gueroult, M. (1930). L'évolution et la structure de la doctrine de la science chez. Fichte (2 Tomos). Paris: Les Belles Lettres.

-Heimsoeth, H. (1931). Fichte. (M. G. Morente, Trad.). Madrid: Revista de Occidente.

Ivaldo, M. (1996). La idea de la filosofía trascendental en la primera Doctrina de la ciencia. En V. López Domínguez (Ed.), Fichte 200 años después (pp. 11-24). Madrid: Editorial Complutense.

-Kabitz, W. (1902). Studien zu Entwicklungsgeschichte der Fichteschen Wissenschaftslehre aus der Kantischen Pbilosopbie. Berlin: Reuter and Reichard.

-Kant, I. (2007). Crítica de la razón pura. (P. Ribas, Trad.). Madrid: Taurus.

(Lauth, 1978:29)- el spinozismo es para él el mejor representante, el más riguroso y coherente, de la posición especulativa que conduce al determinismo universal y que es necesario superar.

17 Este es, ciertamente, el sentido del primer principio tal como aparece en el Fundamento de toda la Doctrina de la ciencia: "Si se piensa explicitar esta autogénesis [Thathandlung en la cúspide de una Doctrina de la ciencia, he aquí los términos en los que tendría que expresarse: El yo pone originariamente de modo absoluto su propio ser" (Fichte, 1975: 18). 
-Kant, I. (1951). Crítica de la razón práctica. (E. Miñana y Villagrasa y M. García Morente, Trad.). Buenos Aires: Ateneo.

-Kant, I. (1902 y ss.) [AK]. Kants gesammelte Werke. Berlín: Preussische/Deutsche Akademie der Wissenschaften.

-Lauth, R. (1978). Spinoza vu par Fichte. Archives de Philosophie, (41): 27-48.

-López Domínguez, V. (1995). Fichte: acción y libertad. Madrid: Ediciones Pedagógicas.

-Nohl, H. (1911). Miscellen zur Fichtes Entwicklungsgeschichte und Biographie. Kant-Studien, 16 (4), 373- 381.

-Philonenko, A. (1991). Traduction et commentaire de la lettre à F.A. Weisshuhn: fragment de 1790. Revue de théologie et de philosophie (41), 229-248.

-Preul, R. (1969). Reflexion und Gefübl: Die Theologie Fichtes in seiner vorkantischen Zeit, Berlin: De Gruyter.

-Schrader, W. (Comp.). (1997). Anfänge und Ursprünge. Zur Vorgeschichte der Jenaer Wissenschaftslehre. Fichte Studien 9. Amsterdam/Atlanta: Rodopi.

-Serrano, V. (2004). Metafísica y filosofía transcendental en el primer Fichte. Valencia: Editorial de la Universidad Politécnica de Valencia.

-Sparn, W. (2001) "Neologie”. En W. Schneiders (Ed.), Lexicon der Aufklärung (pp. 287-288). München: Beck.

-Wildfeuer, A. G. (1997a). Praktische Vernunft und System. Entwicklungsgeschichtliche Untersuchungen zur ursprünglichen Kant-Rezeption Johann Gottlieb Fichtes. Spekulation und Erfahrung. Stuttgart-Bad Cannstaat: Frommann-Holzboog.

-Wildfeuer, A. G. (1997b). Vernunft als Epiphänomen der Naturkausalität. Zu Herkunft und Bedeutung des ursprünglichen Determinismus J. G. Fichtes. En W. Schrader (Comp.), Anfänge und Ursprünge. Zur Vorgeschichte der Jenaer Wissenschaftslehre. Fichte Studien 9 (pp. 61-144). Amsterdam/Atlanta: Rodopi.

Sumario: 1. Religión y deísmo puro; 2. Sentimiento o especulación; 3. La escisión; 4. La Doctrina de la ciencia como reconciliación; Referencias. 